\title{
Practice-centred evaluation and the privileging of care in health information technology evaluation
}

Mary Darking ${ }^{1 *}$, Rachel Anson², Ferdinand Bravo², Julie Davis², Steve Flowers ${ }^{3}$, Emma Gillingham², $^{2}$ Lawrence Goldberg ${ }^{2}$, Paul Helliwell ${ }^{4}$, Flis Henwood', Claire Hudson², Simon Latimer², Paul Lowes ${ }^{2}$ and lan Stirling ${ }^{5}$

\begin{abstract}
Background: Electronic Patient Records (EPRs) and telemedicine are positioned by policymakers as health information technologies that are integral to achieving improved clinical outcomes and efficiency savings. However, evaluating the extent to which these aims are met poses distinct evaluation challenges, particularly where clinical and cost outcomes form the sole focus of evaluation design. We propose that a practice-centred approach to evaluation - in which those whose day-to-day care practice is altered (or not) by the introduction of new technologies are placed at the centre of evaluation efforts - can complement and in some instances offer advantages over, outcome-centric evaluation models.
\end{abstract}

Methods: We carried out a regional programme of innovation in renal services where a participative approach was taken to the introduction of new technologies, including: a regional EPR system and a system to support video clinics. An 'action learning' approach was taken to procurement, pre-implementation planning, implementation, ongoing development and evaluation. Participants included clinicians, technology specialists, patients and external academic researchers. Whilst undergoing these activities we asked: how can a practice-centred approach be embedded into evaluation of health information technologies?

Discussion: Organising EPR and telemedicine evaluation around predetermined outcome measures alone can be impractical given the complex and contingent nature of such projects. It also limits the extent to which unforeseen outcomes and new capabilities are recognised. Such evaluations often fail to improve understanding of 'when' and 'under what conditions' technology-enabled service improvements are realised, and crucially, how such innovation improves care.

Summary: Our contribution, drawn from our experience of the case study provided, is a protocol for practice-centred, participative evaluation of technology in the clinical setting that privileges care. In this context 'practice-centred' evaluation acts as a scalable, coordinating framework for evaluation that recognises health information technology supported care as an achievement that is contingent and ongoing. We argue that if complex programmes of technology-enabled service innovation are understood in terms of their contribution to patient care and supported by participative, capability-building evaluation methodologies, conditions are created for practitioners and patients to realise the potential of technologies and make substantive contributions to the evidence base underpinning health innovation programmes.

Keywords: Electronic patient records, Telemedicine, Practice, Participatory methods, Care, Evaluation, Capability building

\footnotetext{
* Correspondence: m.l.darking@brighton.ac.uk

${ }^{1}$ School of Applied Social Science, Faculty of Health, University of Brighton, Mayfield House, Falmer BN1 9PH, UK

Full list of author information is available at the end of the article
}

\section{Biomed Central}

(c) 2014 Darking et al.; licensee BioMed Central Ltd. This is an Open Access article distributed under the terms of the Creative Commons Attribution License (http://creativecommons.org/licenses/by/2.0), which permits unrestricted use, distribution, and reproduction in any medium, provided the original work is properly credited. 


\section{Background}

Evaluation activities can generate important opportunities for reflection, analysis and intervention. The extent to which they do is a question of evaluation design. In the context of health information technologies (IT) used in hospital care, such as electronic record systems and telemedicine, 25 years of research and implementation effort has shown that predicted outcomes prove hard to achieve in practice [1-4]. Conventional positivist approaches to evaluation which measure cost and clinical outcomes alone lack the scope and flexibility to be able to account for and produce potentially valuable 'lessons learned' from the unintended and unachieved. Those involved in large scale health IT evaluations have proposed a number of alternative guidelines and approaches to inform research and evaluation $[3,5,6]$.

We add to and build upon this body of work by offering a protocol for health IT evaluation that aligns evaluation with changes achieved 'in practice' [7-10] rather than changes that are predicted or envisaged. In line with findings from other significant research on health IT evaluation we argue that a number of recurrent evaluation challenges can be resolved by critically engaging with the questions: who is involved in evaluation and what are they seeking to achieve? Asking these questions presents an opportunity to focus attention on the priorities and concerns of those taking part in implementing and using health IT. In our case study this led to the direct involvement of patients and clinicians and a specific focus on how health IT could bring improvements in care. It also produced a concern with 'where' and 'when' health information technology-related changes materialise as changes to the 'practice of care' $[11,12]$. Having collectively established these evaluation priorities and identified them as 'practice-centred', we went on to ask the question: how can a practice-centred approach be embedded into evaluation of health information technologies? Our particular interest was in how a practice-centred approach could help shift the focus of health IT evaluation towards improvement in patient care.

We found that aligning evaluation efforts to the articulation of how specific capabilities emerged and questions of who is in a position to account for the difference those capabilities have made to the practice of care, offered a framework within which to understand, evaluate and communicate the outcomes of implementation efforts. On the basis of our experience, we make the case that those 'best placed' to comment on and assess technology-related change or lack thereof, are those involved in the work of caring - or 'care practice' as we refer to it - and include healthcare staff, patients and informal carers. We therefore propose that a participatory model of implementation supported by action learning and a programme of collaborative, participatory research can positively influence the outcomes of health information technology implementation and ongoing use. Our contribution, drawn from our experience and the case study provided, is a protocol for practice-centred, participative evaluation of technology in the clinical setting.

\section{The case study: the sussex renal innovation programme (SRIP)}

The Sussex Renal Innovation Programme (SRIP) is a regional programme of technology-enabled innovation in renal services (2009 - ongoing) [13]. The programme was underpinned by the procurement and implementation of a regional EPR system that would support, in addition to many other clinical activities, new telemedicine and teleconferencing capabilities. Medical care for kidney disease is typically organised around a specialist unit (which may or may not include a kidney transplant centre), satellite dialysis units and home dialysis support. The information intensive nature of renal care coupled with a need to provide services across a geographically dispersed population enhanced the need for a 'whole system' approach. Additionally, a significant characteristic of the kidney patient community is that the capacity exists for them to be able to access information from their electronic patient record via an open source technology originating from the renal community called Renal Patient View [14]. The new EPR system would enable all patients in this region to have access to this facility.

A distinctive element of the SRIP was its stated aim and consequent adherence to a 'user-centred' [15] approach which led to the constitution, very early on, of a multi-disciplinary, patient-involved action learning group called the 'SRIP Action Learning Group' (ALG) [16]. Membership of the ALG was by invitation of the clinical lead nephrology consultant for the SRIP. A typical meeting would include a Ward Nurse Manager, a Nurse Specialist, the Renal Data Manager, an Administration Manager, the Haemodialysis Unit Manager, a Change Facilitator, a Practice Educator, a Social Care expert, a junior doctor on the renal unit and a Patient Representative [17].

The ALG began meeting during the strategic development of the programme and these discussions informed the EPR procurement process. The EPR procurement process was carried out participatively with all ALG members invited to attend product demonstrations and engage in discussions with potential suppliers. A specialist, renal EPR system was purchased and in addition, a data analytics module was commissioned along with two 'integration pieces': one that would feed results from regional microbiology and virology laboratories into the EPR; and one that would link the EPR to the patientfacing portal Renal Patient View. The video clinics and teleconferencing component was initially progressed as a 
pilot project by a small sub-group of the ALG working in collaboration with a local, university-based innovation centre who provided the necessary technical equipment and expertise [18].

\section{Methods: developing a participative, practice- centred approach}

From its inception, the ALG invited 'outsider' researchers and health policy specialists to group meetings in order to bring alternative perspectives to discussions. A research team from a local university was invited to join the programme on a 30 day consultancy contract to be carried out over a 6 month period between April 2010 and October 2010. It was assumed that this time period would include pre-implementation planning, implementation itself and allow one month for post-implementation observation. Having stated their intention to be 'partners in innovation', representatives of the EPR supplier also became active members of the ALG attending meetings from April 2010.

EPR and telemedicine implementations are widely acknowledged as being complex and contingent areas of technology-enabled service innovation [5,6]. Whilst broad outcome measures for the SRIP were defined in advance the dynamic and evolving character of events meant specific benefits were hard to pinpoint due to the intensive product development, technology infrastructure work, hardware procurement and implementation activities taking place [6].

Inevitably, the EPR implementation over-ran and 'go live' was delayed from September 2010 to December 2010. This altered the original implementation evaluation design which was scheduled to finish after 6 months. By mutual agreement the evaluation period was extended so that the university team could see what changes to practice the EPR implementation effort would finally produce. Ongoing ALG meetings, a super user strategy and cascade training model ensured that clinical staff were prepared for 'go live' and that adequate support would be available 'on the shop floor'.

As is often the case, the integration aspects of the programme took longer than expected and so benefits associated with Renal Patient View and the integration of laboratory results were delayed. Similarly, 'go live' activities focused on the main unit in the first instance with satellite units scheduled to follow at a later date. Within the main unit the decision had been taken that the ward would not go live at this point due to concerns that it would lead to 'dual administrative systems' (i.e. paper and electronic) which ward nurses contended would impact on time available for direct patient care [19]. Nonetheless, for the remaining areas of the renal unit the clinical team, technology supplier, data manager and administrative staff achieved an impressive 'business as usual' switch to the new system (apart from an issue affecting the production of patient letters for which a workaround had to be found).

Initially, in the weeks following 'go live' it appeared that 'nothing had changed'. In many respects this was an indication that the implementation had been a success, but in other respects it was disappointing not to see immediate benefits. Process mapping in the months preceding implementation had identified specific opportunities for achieving service efficiencies through use of the EPR. However, it was clear that these planned improvements would require significant service innovation effort on the part of staff and would take up a substantial amount of time and effort. As those involved in the SRIP were already contributing a considerable amount of time to implementation activities, which in turn were taking longer than predicted, the timescale for these planned improvements had to move from pre-implementation to postimplementation. However, unplanned improvements did occur fairly soon after implementation. For example, on seeing how the system worked in situ, an ALG member and nurse manager identified a means through which the new system could support 'named nurse meetings' and was able to act on this almost immediately. Through encountering these examples ALG members became conscious that the benefits of the system would not 'just happen' post-implementation but would have to be pushed through by nursing and medical staff.

From this point, it was nursing staff who progressed the ongoing development of the EPR system and postimplementation benefits realisation, with focussed, strategic input from the lead consultant. Although another consultant and a hospital registrar would attend ALG meetings occasionally, the group was Chaired and predominantly attended by nurses. Nurses took on leadership roles in key parts of the programme delivery such as training, technical support and hardware procurement/trialling [20]. They became very knowledgeable about the EPR product, issues that affected system use and data quality, and how to address these issues through negotiation with the supplier [21]. They instituted processes for: tracking 'change requests' and 'bug fixes'; held the supplier to account for slippages in the implementation timeframe; and insisted that the product developers responded to needs arising from opportunities they identified for EPR-supported service improvement. Involvement of supplier representatives as members of the ALG and the capacity this created for direct, face-to-face interaction, enhanced supplier responsiveness, encouraging clinician engagement.

The EPR implementation occupied so much staff time that video clinics were not piloted until 8 months after the EPR went live. Two clinics with 4 patients in each were successfully piloted [18]. The hardware used for the pilot did not belong to the Trust and so a procurement process 
was entered into with local trust information services. Procurement and subsequent trialling processes were still ongoing as of March 2013.

The external evaluation team, originally contracted to evaluate the EPR system implementation, remained engaged during this post-implementation period with one member now acting as a participant researcher within the ALG. With numerous post-implementation issues to resolve it became clear that knowing 'when' the system would bring a change to practice and who within the broader renal clinical team would enact that change would be vital $[22,23]$. A 'watch list' of benefits that were 'on the horizon but not here yet' was constructed. It was difficult to attach precise timeframes to 'when' benefits would be realised as work was still very much ongoing. Four years on from implementation several key pieces of the original implementation plan remained uncompleted, such as the integration of blood test results from regional laboratories with the EPR. Significant efficiencies and patient benefits could be realised from this element of the programme. However, the multi-stakeholder nature of this element and the need for input from institutional level IT departments has resulted in slow progress.

\section{Key findings}

It is well-documented that programmes of innovation in service delivery that involve telemedicine and EPR system implementation present distinct evaluation challenges due to their typically dynamic and evolving nature $[24-26,5,6]$. Our experience of the SRIP supports these findings. However, whilst the programme did not proceed according to pre-specified timescales and outcome measures, the participative and practicecentred approach to evaluation enabled alternative guiding principles to emerge. These principles were unified by our co-participants' over-riding concern with care and how it is practiced. We embedded this concern in our approach and refer to it as 'privileging care'. We discuss these guiding principles and the privileging of care below before going on to show how they can form the basis of a protocol for future capability building evaluation research.

\section{Competing demands and the defence of 'time to care'}

Clinical practitioners had limited time to commit to evaluation activities. This response was partly due to a broader climate of perceived 'over-audit' and partly due to the significant time that implementation activities were taking up [27]. Clinical staff were actively engaged in collecting and collating a range of information to meet business, clinical and corporate requirements. From their perspective this data was 'sent out' of the unit and where results were returned they formed part of annual or quarterly feedback cycles that did not improve the information held on patients for whom they were currently responsible [28]. Clinicians with management and leadership responsibilities expressed similar concerns regarding a lack of information to reflect the immediate, day-to-day organisation of services. This state of affairs is represented in Figure 1 as a simple data flow model. In this model, clinical practice, care and learning are regarded as fundamental activities that are nonetheless perceived as 'one step removed' from day-to-day system use and data quality. In this model the purpose of the EPR system is understood to be more closely allied to business and cost reporting, clinical audit and corporate, Trust-wide requirements than care, clinical practice and learning.

ALG members were of the unanimous opinion that the EPR system had to lessen rather than add to the audit and administration burden, releasing time for direct patient care, or else it would be unusable. This was particularly pertinent in the case of ward nursing in the main renal unit [19]. There was also consensus that the system should support 'better care' and 'learning' about what 'helped patients' first and foremost, and that other reporting demands should be served as a consequence of meeting those aims.

\section{Privileging care}

The priorities expressed by ALG members concerning what they hoped to achieve from the SRIP guided implementation planning and informed the emerging framework for evaluation. 'Privileging care' described an aspirational

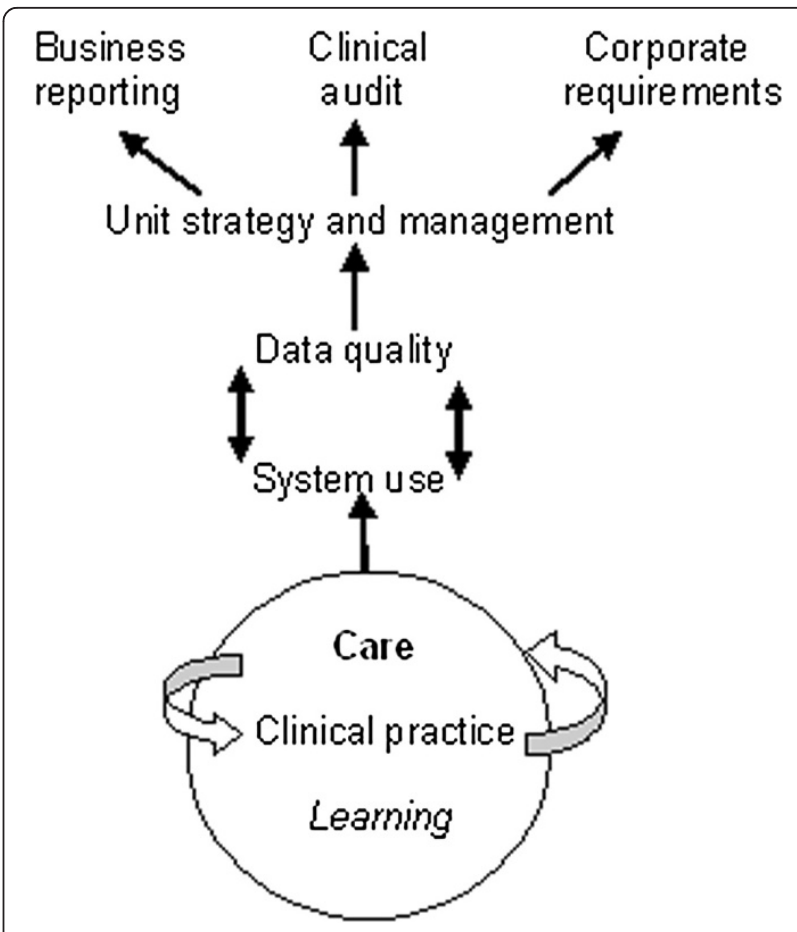

Figure 1 Existing data flow model. 
position whereby new technology would enhance care by creating opportunities to reflect upon and improve clinical practice, and support patient well-being. In an ideal scenario, system use and data quality would relate directly to these goals and other data needs met as a consequence of serving these ends. In this sense, other needs were not only those related to clinical audit, business reporting and corporate requirements for information, but also greater recognition of the interdisciplinary use of data across the clinical team. Sharing best practices and insights gained into patient care through use of the system in itself becomes a source of learning and of new ideas for service innovation. With improvements in data quality, EPR data also become available for use in staff research projects encouraging a culture of research around health IT use that supports ongoing development. Figure 2 describes this position in terms of an ideal model for data generation and use.

\section{Patient involvement}

Patient involvement in the ALG altered the way in which care and evaluation were conceived of and discussed. Developing an ongoing model of evaluation that assumed the involvement of patients brought about a redefinition of what kinds of care were significant in the context of the EPR. Having the patient representative participating in the ALG broadened the notion of what constitutes renal care and who is involved in that care. Conventionally, within healthcare contexts, care is defined in terms of medical and clinical practice, i.e. professional care-giving. These constructions can conceal the work that patients and informal carers put into coping with illness and potentially obscure the ways in which patients are active in their own 'self' care [29,30,31].

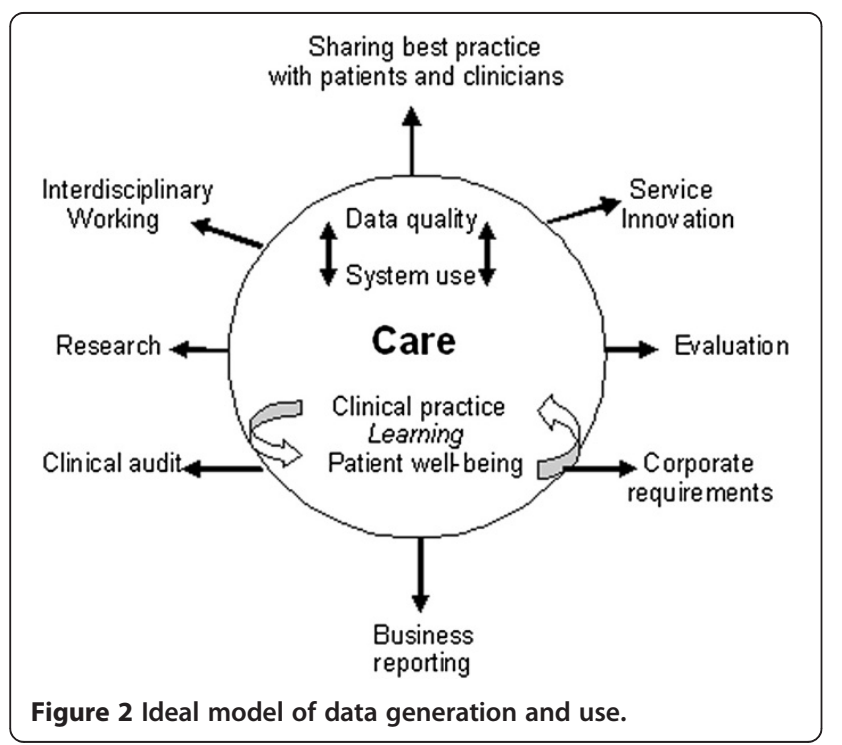

In the development of the evaluation methodology this provoked a shift from understanding practice as alluding specifically to 'medical' or 'clinical practice' to 'care practice' defined as knowledgeable activities engaged in as part of everyday routines associated with both formal and more informal forms of care $[11,19,32]$.

\section{Carer roles}

The kidney patient representative routinely emphasised the wider network of care and, in particular, the significance of carer involvement in patient care [32,33]. For renal patients, the involvement of family members, friends or other representatives can be crucial, in some cases determining whether or not a patient can consider home dialysis options. For patients' carers, having (via patient consent) access to information that can support informal care activities offers significant benefits. A NHS Kidney Care evaluation of Renal Patient View [13] analysed usage statistics which showed that use of Renal Patient View increased when kidney patients experienced acute episodes and were admitted as in-patients. Given that many hospital wards do not offer wireless internet access and that most inpatients have immediate access to their blood results via their nurse or consultant, the conclusion drawn by the evaluation was that it was carers logging onto the system from home for updates that caused a spike in usage statistics.

\section{Invisibility of systems to stakeholders}

As with most care record systems, it is healthcare professionals rather than patients who are typically understood as 'users' of the system. Patients are likely to never see, nor will they ever directly input, data into their hospital's EPR system, yet that system will form a cornerstone of their care. This creates a potential barrier to patient and public understanding of ethical considerations and potential safety issues that might arise from EPR and biomedical data use $[34,35]$. In terms of patient EPR awareness, this situation is slightly different for renal patients living in areas where Renal Patient View is available. In these cases, all patients, carers and GPs have the opportunity to see a view of the EPR data and therefore act on the basis of new test results [13]. For the patient representative in our example, this made community engagement in the implications and outcomes of the EPR implementation more compelling and tangible, translating a complicated and remote institutional IT change into an issue of immediate relevance to patients and questions of care [36]. It also altered the evaluation approach by increasing the scope for involving patients in evaluation data collection efforts and crucially, in evaluation design.

This model expresses a desire for a direct and positive association between system use and patient care, based 
on a timely feedback cycle where patient data is central to ongoing processes of learning, service improvement and patient education. The antithesis of this model that EPR system use would increase time spent on data entry and administration without releasing time to care - was positioned by participants as not just undesirable but untenable. The question of whether EPR system use would lessen administration and create more opportunities to review patient information in real time, remained a central concern throughout the implementation and ongoing development process [19].

\section{Implications: a protocol for participative, practice-centred evaluation that privileges care}

The external researchers involved in coordinating evaluation efforts identified generalizable principles from the case study described above. These principles were informed theoretically by the notion of 'practice', which places a priori emphasis on what it is that people do, from day-to-day, in specific contexts and settings. A practice-centred approach to evaluation is supported by a participative approach to evaluation design, data collection and analysis because one of its primary aims is to identify and engage those people 'best placed' to see 'when' and 'how' technology-related changes materialise. This process is emergent and ongoing because it is accepted at the outset that the outcomes of health IT implementations are cannot be fully known in advance. Taking this position has allowed us to identify and evaluate examples of: changes to practice that had been predicted; changes to practice that had been predicted but which did not proceed as expected; changes to practice that did not materialise; and changes that occurred as the result of unanticipated, innovative interactions between the system and those seeking ways to incorporate it into their care practice.

Table 1 provides practical guidelines for designing and undertaking a practice-centred evaluation. Our approach incorporates both practice-centred and participative elements and so the table below provides guidelines that refer to both elements respectively. Whilst a practicecentred evaluation does not necessarily require a participative approach to evaluation activities, we found that the two were very closely linked. Therefore, in the table below, the left-hand column describes how to design evaluation activities so that they are practice-centred and in this case, privilege care. In addition, the righthand column highlights ways to support participative evaluation work and promote critical reflection on the nature of and extent to which participation is achieved.

Using this approach, SRIP evaluators have so far conducted evaluations using: 'before' and 'after' evaluation data;

\section{Table 1 A protocol for practice-centred evaluation that privileges care}

\section{Practice-centred \\ - Organise evaluation around care 'scenarios' where EPR related change} has (or has not) occurred

- Expect opportunistic or emergent change to be generative of significant benefits and problems

\footnotetext{
- Develop an evaluation 'watch list' of areas of practice where it is anticipated EPR related changes to practice will occur in order to capture if/when those changes occur

- Include in this list: change anticipated as part of specific service improvement activities; and/or change relating to EPR functionalities that have been either speculated upon and/or specified in advance; and/or potential negative changes to practice

- Capture 'before change' data relevant to both practice and outcomes, where possible or relevant

- Wait for change to materialise 'in practice' before collecting 'after change' data

- Actively include care scenarios where EPR related changes have: not proved possible; only partially been achieved; required an unanticipated amount of effort; proven exceptionally slow to achieve; or proven unachievable
}

Participative

- Organise evaluation activities around core values and priorities of those participating. In our case: patient wellbeing, learning and care

- Encourage those experiencing or producing health information technology-related changes to participate in designing and where possible carrying out evaluation of those changes

- Work collaboratively in groups including at least one person who is directly involved in the change being evaluated

- Use multiple data sources (e.g. questionnaire data, system usage data, interviews), admit diversity of opinion and resist synthesis or judgement

- Encourage collective reflection on the boundary of involvement in evaluation (i.e. 'who' or 'what' is included/excluded) and seek ways to counter persistent exclusion

- Report findings in a way(s) that 'speaks' to the community of practice concerned 
case study based description; and patient questionnaires $[37,38,18]$. They have reported their findings at conferences attended by clinical colleagues, nurse research seminars within the hospital trust, at a research symposium to designed to stimulate interest from local universities in secondary care informatics and at a national user group meeting they coordinated in collaboration with their EPR supplier.

\section{Future research agenda}

We have argued the case for a participative, practicecentred approach to health information technology evaluation that privileges care. We have done so on the basis that such an approach supports the production of evidence pertaining to how, when and for whom health information technology systems can be said to change care and care practice. Based on our experience, we argue that patient involvement and nurse leadership is likely to be at the forefront of efforts to realise health information technology benefits and ensure these benefits are oriented toward better care for patients. Supporting nurses in this role will require capability building in research and evaluation, which in turn requires the recognition and endorsement of these skills in programmes of education and professional development. There is potential for participative, action learning-based evaluation efforts to support the development of health IT knowledge within and across hospital trusts. We argue that this does not just support shared understanding but also the 'in practice' realisation of health IT and its potential. This particular finding holds implications for health IT producers and hospital Trust strategic leads as much as it does patients, carers, clinicians and the general public. From our experience, there is clear enthusiasm for sharing experience that can and should be mobilised. Four years after 'go live', the ALG is still meeting and is keen to share what they have learned with clinical groups participating in the hospital-wide EPR implementation taking place in their hospital trust and other users of renal EPR systems.

\section{Competing interests}

The authors declare that are no competing interests.

\begin{abstract}
Authors' contributions
The ideas presented here emerged from collaborative discussions held in the SRIP Action Learning Group (ALG). All authors were members of the ALG apart from SF and FH who (along with MD) formed the university evaluation team. Early versions of the text were presented as summative feedback to the ALG where the text, ideas and arguments were commented on extensively. RA, FB, SF, EG, LG, PH, FH, CH, PL and IS all contributed to several revisions of the manuscript and the intellectual development of the article. All authors read and approved the final manuscript.
\end{abstract}

\section{Acknowledgements}

We are grateful to all past and present attendees of the SRIP Action Learning Group (who are not listed as authors) for their contribution to the intellectual development of this methodology. In particular we would like to recognise the contribution of Professor Bob Sang who previously held the position of Chair of Public and Patient Involvement at London South Bank University. We are grateful to Brighton and Hove City Primary Care Trust for funding the SRIP pilot and to Candy Morris, NHS South of England for her support in shaping the SRIP strategic vision. We would also like to recognise the contributions of Lynn Smith, Programme Lead for Benefits Realisation \& EDT from the Strategic Health Authority South East and Toto Grunland, Department of Health, Public and Patient Involvement Lead. Finally, we would like to thank all staff and patients at Sussex kidney and dialysis units whose experiences and concerns have informed this work.

\section{Funding}

This article reports on an evaluation that was commissioned by the Brighton and Hove City Primary Care Trust. The views expressed in this publication are those of the authors and not necessarily those of the NHS, the Brighton and Hove City Primary Care Trust or the Department of Health.

\section{Author details}

${ }^{1}$ School of Applied Social Science, Faculty of Health, University of Brighton, Mayfield House, Falmer BN1 9PH, UK. ${ }^{2}$ Sussex Kidney Unit, Royal Sussex County Hospital, Brighton and Sussex University Hospitals NHS Trust, Eastern Road, Brighton, BN2 5BE, UK. ${ }^{3}$ Centre for Innovation Management (CENTRIM), Freeman Centre, University of Brighton, University of Sussex Campus, Falmer BN1 9QE, UK. ${ }^{4}$ Clinical Computing Limited, 1 Bath Street, Ipswich IP2 8SD, UK. ${ }^{5}$ South Eastern Kidney Patients Association (SEKPA), c/o Sussex Kidney Unit, Royal Sussex County Hospital, Brighton and Sussex University Hospitals NHS Trust, Eastern Road, Brighton BN2 5BE, UK.

Received: 25 March 2013 Accepted: 2 May 2014

Published: 5 June 2014

\section{References}

1. Berg M: Implementing information systems in health care organizations: myths and challenges. Int J Med Inform 2001, 64(2-3):143-156.

2. Jones M: 'Computers can land people on Mars, why can't they get them to work in a hospital?': implementation of an electronic patient record system in a UK hospital. Methods Inf Med 2003, 42(4):410-415.

3. Takian A, Petrakaki D, Cornford T, Sheikh A, Barber N: Building a house on shifting sand: methodological considerations when evaluating the implementation and adoption of national electronic health record systems. BMC Health Serv Res 2012, 12:105.

4. Elwyn G, Hardisty A, Peirce S, May C, Evans R, Robinson D, Bolton C, Yousef Z, Conley E, Rana O, Gray WA, Preece AD: Detecting deterioration in patients with chronic disease using telemonitoring: navigating the 'trough of disillusionment'. J Eval Clin Pract 2012, 18(4):896-903.

5. Greenhalgh T, Russell J: Why do evaluations of eHealth Programs fail? An alternative set of guiding principles. PLOS Med 2010, 7:11.

6. May C, Finch T, Ballini L, MacFarlane A, Mair F, Murray E, Treweek S, Rapley T: Evaluating complex interventions and health technologies using normalization process theory: development of a simplified approach and web-enabled toolkit. BMC Health Serv Res 2011, 11:245.

7. Fenwick T: Sociomateriality in medical practice and learning: attuning to what matters. Med Educ 2014, 1(1):44-52.

8. Lave J, Wenger E: Situated Learning: Legitimate Peripheral Participation (Learning in Doing: Social, Cognitive and Computational Perspectives). Cambridge: Cambridge University Press; 1991.

9. Orlikowski WJ: Using technology and constituting structures: a practice lens for studying technology in organizations. Organ Sci 2000, 11(4):404-428.

10. Greenhalgh T, Swinglehurst D: Studying technology use as social practice: the untapped potential of ethnography. BMC Med 2011, 9:45.

11. Mol A, Moser I, Pols J: Putting practice into theory. In Care in Practice: on Tinkering in Clinics, Homes and Farms. Edited by Mol A, Moser I, Pols J. Beilefeld: Transcript Verlag; 2010:7-26.

12. Barad K: Getting Real: Technoscientific Practices and the materialization of reality. In Meeting the Universe Halfway: Quantum Physics and the Entanglement of Matter and Meaning. Edited by Barad K. London: Duke University Press; 2007:189-222.

13. Goldberg L, Pollak S: A patient-centric approach to computerising disease management across sussex: specifying a regional pilot to personalise the 
total care of patients with kidney disease: an initial analysis of costs and benefits. Report to Primary Care Trust 2008, 1-36.

14. Mukoro F: Renal patient view: a system which provides patients online access to their test results, Final evaluation report. London: NHS Kidney Care; 2012.

15. Flowers S: New directions in user innovation research: the developing policy research agenda. In Perspectives on User Innovation, Series on Technology Management, Volume 16. Edited by Flowers S, Henwood F. London: Imperial College Press; 2009.

16. Department of Health: The Renal Action Learning Sets Phase II Interim Report. London: 2007. Ref 8464.

17. Goldberg L: How can information technology work for wellbeing? Dialogue, data and digital media. In Better health in harder times: active citizens and innovation on the frontline. Edited by Walmsley J, Davies C, Hales M, Flux R. Bristol: Policy Press; 2012.

18. Anson R, Pearson S, Bissenden A, Goldberg L: Piloting videotelephony-assisted renal clinics: Initial experience and lessons learnt, British Renal Society Conference; 2012 May 1-3; Manchester. 2012. Available from URL: http:// www.britishrenal.org/Conferences/Conferences-Home/BRS-Conference2012/Poster-Programme-2012.aspx.

19. Darking M, Flowers S, Gillingham E, Goldberg L, Henwood F: Care, technology and administration: an ethic of care analysis of the competing demands placed on ward nursing. In In the Proceedings of Critical Care. Brighton: 2012.

20. Tomlinson J: Exploration of transformation and distributed leadership. Nursing Manage 2012, 19(4):30-34.

21. Michel-Verkerke MB: Information quality of a nursing information system depends on the nurses: a combined quantitative and qualitative evaluation. Int J Med Inf 2012, 81:662-673.

22. Pollock N: When is a workaround? Conflict and negotiation in computer system development. Sci Technol Hum Values 2005, 30(4):1-19.

23. Ellingsen G, Monteiro E, Roed K: Integration as interdependent workaround. Int I Med Inform 2012, in press.

24. Heathfield H, Pitty D, Hanka R: Evaluating information technology in health care: barriers and challenges. Br Med J 1998, 316:1959-1961

25. Ammenwerth E, Graber S, Herrmann G, Burkle T, Konig J: Evaluation of health information systems - problems and challenges. Med Inf 2003, 71:125-135.

26. Littlejohns P, Wyatt J, Garvican L: Evaluating computerised health information systems: hard lessons still to be learnt. Br Med J 2003, 326(7394):860-863.

27. Bowie P, Bradley N, Rushmer R: Clinical audit and quality improvement - time for a rethink? J Eval Clin Pract 2012, 18(1):42-48.

28. Haynes P: Chaos, complexity and transformations in social care policy in England. Public Money Manage 2007, 3:199-206.

29. Akrich M: From communities of practice to epistemic communities: health mobilizations on the internet. Sociol Res Online 2010, 15:2.

30. Callon M, Rabeharisoa V: The growing engagement of emergent concerned groups in political and economic life. Lessons from the French Association of neuromuscular disease patients. Sci Technol Hum Values 2008, 33(2):230-261.

31. Rogers A, Kirk S, Gately C, May CR, Finch T: Established users and the making of telecare work in long term condition management: implications for health policy. Soc Sci Med 2011, 72(7):1077-1084.

32. Barnes M: Care in everyday life: an ethic of care in practice. Bristol: Policy; 2012

33. Morton RL, Tong A, Howard K, Snelling P, Webster AC: The views of patients and carers in treatment decision making for chronic kidney disease: systematic review and thematic synthesis of qualitative studies. Br Med J 2010, 340:7742.

34. Bratan T, Stramer K, Greehalgh T: 'Never heard of it' - understanding the public's lack of awareness of a new electronic patient record. Health Expect 2010, 13(4):379-391.

35. Hurdle JF, Adams SA, Brokel J, Chang B, Embi PJ, Petersen C, Terrazas E, Winkelstein P: A code of professional ethical conduct for the American Medical Informatics Association: an AMIA Board ofDirectors approved white paper. J Am Med Inform Assoc 2007, 14(4):391-393.

36. Taylor M, Karet Frankl FE: Developing a strategy for the management of rare diseases: needs central coordination and input from patients. BMJ 2012, 324(e2417):344-345.

37. Anson R, Bravo F, Darking M, Helliwell P, Goldberg L: Implementing the Sussex Renal Innovation Programme: implications for outpatient service delivery, British Renal Society Conference. Manchester: 2012. Available from URL: http://www.britishrenal.org/Conferences/Conferences-Home/BRSConference-2012/Poster-Programme-2012.aspx.

38. Hudson C, Stirling I, Davis J, Anson A, Goldberg L: Using Renal patient view to improve the patient experience - measuring the value of the information patients receive, British Renal Society Conference. Manchester: 2012. Available from URL: http://www.britishrenal.org/Conferences/ConferencesHome/BRS-Conference-2012/Poster-Programme-2012.aspx.

doi:10.1186/1472-6963-14-243

Cite this article as: Darking et al:: Practice-centred evaluation and the privileging of care in health information technology evaluation. BMC Health Services Research 2014 14:243.

\section{Submit your next manuscript to BioMed Central and take full advantage of:}

- Convenient online submission

- Thorough peer review

- No space constraints or color figure charges

- Immediate publication on acceptance

- Inclusion in PubMed, CAS, Scopus and Google Scholar

- Research which is freely available for redistribution 